\title{
Rapid Degradation of Cellulose Diacetate by Marine Microbes
}

\author{
Michael G. Mazzotta,* Christopher M. Reddy, and Collin P. Ward* \\ Cite This: Environ. Sci. Technol. Lett. 2022, 9, 37-41 \\ Read Online
}

ABSTRACT: The persistence of cellulose diacetate (CDA), a biobased plastic used in textiles and single-use consumer products, in the ocean is currently unknown. Here, we probe the disintegration and degradation of CDA-based materials ( $25 \mu \mathrm{m}$ films, $510 \mu \mathrm{m}$ foam, and $97 \mathrm{~g} / \mathrm{m}^{2}$ fabric) by marine microbes in a continuous flow seawater mesocosm. Photographic evidence and mass loss measurements demonstrate that CDA-based materials disintegrate in months. Disintegration is marked by the increasing esterase and cellulase activity of the biofilm community, suggesting that marine microbes degrade CDA. The natural abundance stable $\left({ }^{13} \mathrm{C}\right)$ and radiocarbon $\left({ }^{14} \mathrm{C}\right)$ isotopic signature of carbon dioxide respired during short-term bottle incubations confirms the rapid degradation of both acetyl and cellulosic

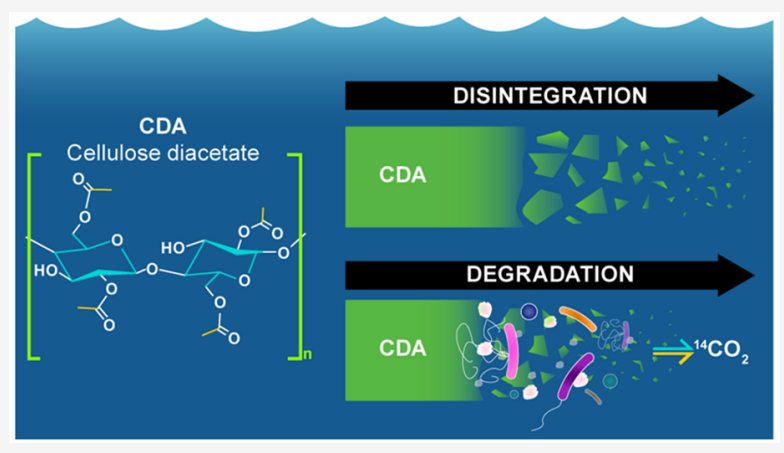
components of CDA by seawater microbial communities. These findings challenge the paradigm set by governmental agencies and advocacy groups that CDA-based materials persist in the ocean for decades, and represent a positive step toward identifying highutility, biobased plastics with low environmental persistence.

\section{INTRODUCTION}

The ubiquitous presence of plastic debris in the world's oceans, ${ }^{1}$ alongside exponentially growing consumer demand for plastic, ${ }^{2}$ necessitates the identification of sustainably sourced plastics with low persistence in marine environments. Cellulose diacetate (CDA) is a primarily biobased (derived from wood pulp $^{3}$ ) plastic that is widely used in consumer goods, including textiles, coatings, films, and other high-use products. By far, single-use, CDA-based cigarette filters are the most prevalent form of marine debris in the world. ${ }^{4}$ The assumption by the National Oceanic and Atmospheric Association (NOAA), international governmental agencies, and advocacy and media groups is that CDA-based materials persist in the oceans for up to a decade. ${ }^{5,6}$ However, while CDA appears to be labile to microbial attack in soil and wastewater environments, ${ }^{7,8}$ no peer-reviewed, environmentally relevant study has assessed the persistence of CDA-based materials in marine environments.

Here, we incubated CDA-based materials and positive (high degradative capacity) and negative (low degradative capacity) control materials in a mesocosm equipped with a continuous flow of coastal seawater (Vineyard Sound, Massachusetts, United States; see the Supporting Information for experimental details). Four formulations of CDA were tracked over time in the mesocosm, including nonplasticized and plasticized (triacetin) CDA $25 \mu \mathrm{m}$ films, a plasticized (triacetin) 510 $\mu \mathrm{m}$ CDA foam, and a nonplasticized CDA fabric $\left(97 \mathrm{~g} / \mathrm{m}^{2}\right)$. As positive controls, ${ }^{9}$ we tracked Kraft paper film and cotton fabric, both cellulosic in nature. As negative controls, ${ }^{10-12}$ we tracked a polyethylene (LDPE) film and polyethylene terephthalate (PETE) fabric. Time-lapse photography and mass loss measurements documented the disintegration of these materials. Enzymatic assaying and analysis of the natural abundance radiocarbon $\left({ }^{14} \mathrm{C}\right)$ and stable carbon $\left({ }^{13} \mathrm{C}\right)$ isotopic composition of the carbon dioxide $\left(\mathrm{CO}_{2}\right)$ respired during short-term bottle incubations documented biodegradation of these materials. Our collective findings demonstrate that CDAbased materials disintegrate and biodegrade in the ocean orders of magnitude faster (months) than previously reported (decades).

\section{MATERIALS AND METHODS}

This study uses several orthogonal yet complementary analytical techniques to track the disintegration and biodegradation of CDA-based materials and positive and negative control materials in the ocean. Complete descriptions of the experimental details and analytical approaches are available in the Supporting Information (Sections S1-S15).

The physical and chemical properties of CDA materials (25 $\mu \mathrm{m}$ films, $510 \mu \mathrm{m}$ foam, and $97 \mathrm{~g} / \mathrm{m}^{2}$ fabric), positive control materials (100 $\mu \mathrm{m}$ cellulose film and $91 \mathrm{~g} / \mathrm{m}^{2}$ cotton fabric), and negative control materials (25 $\mu \mathrm{m}$ LDPE film and $126 \mathrm{~g} /$ $\mathrm{m}^{2}$ PETE fabric) are described in Sections S1 and S2. These

Received: October 18, 2021

Revised: November 29, 2021

Accepted: November 29, 2021

Published: December 8, 2021 
materials were incubated in a custom-built mesocosm with a continuous flow of Vineyard Sound seawater at $20{ }^{\circ} \mathrm{C}$ (Section S4 and Figure S1). In total, nearly $350 \mathrm{CDA}$ and control samples were incubated in the mesocosm throughout the 25week experiment.

At specified time points, CDA and control materials were photographed to visually assess disintegration (Figure 1 and

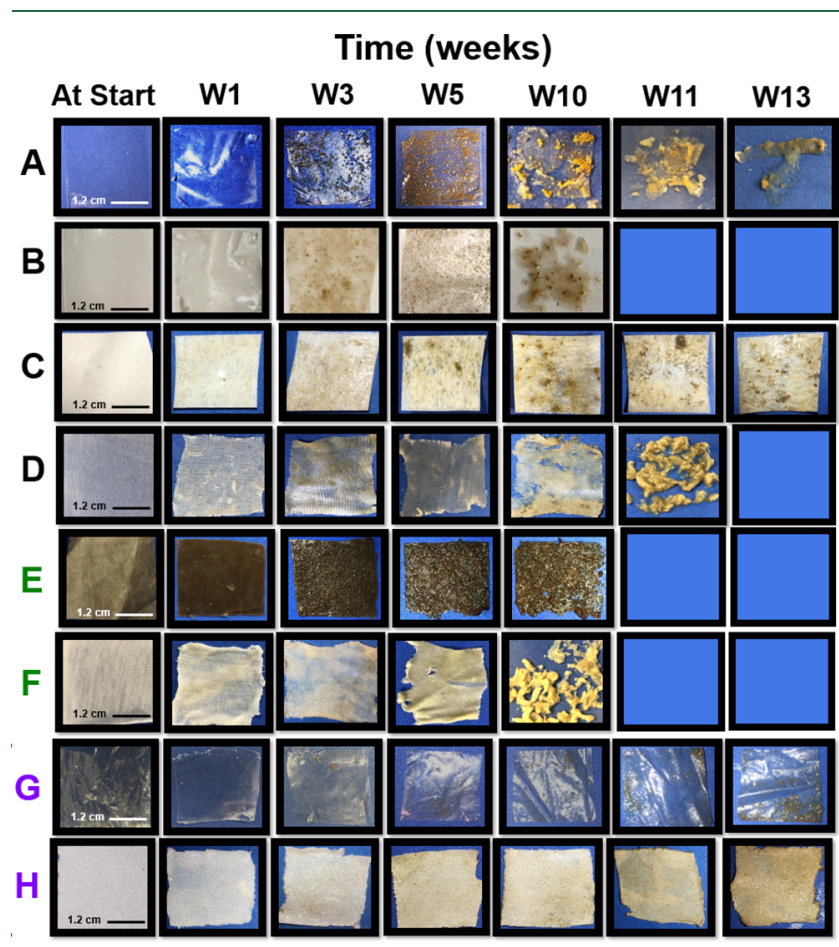

Figure 1. Time-lapse photography showing visual disintegration of certain materials (CDA and positive controls) and not others (negative controls) over a 25-week incubation in a continuous flow seawater mesocosm (week 25 photographs in Figure S2). Blue-filled boxes represent complete disintegration. Legend: (A) $25 \mu \mathrm{m} \mathrm{CDA}$ film (no plasticizer), (B) $25 \mu \mathrm{m}$ CDA film (triacetin), (C) $510 \mu \mathrm{m}$ CDA foam, (D) $97 \mathrm{~g} / \mathrm{m}^{2}$ CDA fabric, (E) $100 \mu \mathrm{m}$ Kraft paper, (F) 91 $\mathrm{g} / \mathrm{m}^{2}$ cotton fabric, (G) $25 \mu \mathrm{m}$ LDPE film, and (H) $126 \mathrm{~g} / \mathrm{m}^{2}$ PETE fabric.

Figure S2). To determine the mass that was lost during the incubation, we developed a novel protocol to completely remove biofilm biomass from the CDA and control materials (Section S5 and Figure S3). Removal was accomplished by lysing the cells in deionized water and gently sonicating in a water bath. The protocol was validated using two lines of evidence: staining for adhered cellular biomass with crystal violet and natural abundance radiocarbon analysis (Sections S6 and S7). After complete removal of the biofilm, the CDA and control materials were dried and weighed to determine mass loss over time $[N=4$ (Section S8)].

Fluorophore-based enzymatic assaying was used to profile the esterase and cellulase activities of the natural marine biofilms growing on CDA and control materials (Section S9). At specified time points, intact biofilms growing on CDA and control materials were liberated using bath sonication. The liberated biofilms were then transferred to 96-well plates along with the fluorophore probes to monitor enzymatic activity $(N$ = 3). Esterase activity was monitored using 7-acetoxy-4methylcoumarin, whereas cellulase activity was monitored using the Enz-Chek cellulase reagent (Section S9).
The natural abundance radiocarbon content (expressed as $\Delta^{14} \mathrm{C}$ ) and ${ }^{1} \mathrm{H}$ nuclear magnetic resonance spectroscopy (NMR) tracked compositional changes of CDA residues over time in the continuous flow mesocosm (Sections S10S13). Biomass free CDA materials (Section S5) were analyzed for $\Delta^{14} \mathrm{C}$ using elemental analysis at the National Ocean Sciences Accelerator Mass Spectrometry (NOSAMS) facility. The degree of substitution of CDA residues was determined using a Bruker (Billerica, MA) Avance III HD $600 \mathrm{MHz}$ instrument, equipped with a $5 \mathrm{~mm}$ BBFO Z-gradient SmartProbe.

Lastly, shifts in the $\Delta^{14} \mathrm{C}$ and $\delta^{13} \mathrm{C}$ of the dissolved inorganic carbon ( $\mathrm{DI}^{14} \mathrm{C}$ and $\mathrm{DI}^{13} \mathrm{C}$, respectively) during short-term bottle incubations assessed if native marine microbes respire $\mathrm{CDA}$ and control materials to carbon dioxide $\left[\mathrm{CO}_{2}\right.$ (Sections S14 and S15)]. There were four experimental treatments: (i) seawater only, (ii) CDA and seawater, (iii) cellulose (positive control material) and seawater, and (iv) LDPE (negative control material) and seawater. After 10 weeks in the continuous flow mesocosm, the test materials were transferred to $125 \mathrm{~mL}$ respirometry bottles and filled with $100 \mathrm{~mL}$ of nutrient-amended seawater to exclude the possibility of oxygen and nutrient limitation during the 6-day incubation. Incubations took place in the dark at $20{ }^{\circ} \mathrm{C}$ on a shaker table set at $15 \mathrm{rpm}$. At set time points $(0,2,4$, and 6 days for the CDA treatment and 0 and 6 days for the control treatments), the incubation was ceased with a spike of a saturated $\mathrm{HgCl}_{2}$ solution. At the end of the experiment, all samples were submitted to NOSAMS for $\mathrm{DI}^{13} \mathrm{C}$ and $\mathrm{DI}^{14} \mathrm{C}$ analysis. Isotopic mass balance equations were used to determine the amount and source of $\mathrm{C}$ respired to $\mathrm{CO}_{2}$ by the native microbial communities during the incubation (Section S14).

\section{RESULTS AND DISCUSSION}

Photographic and Mass Loss Evidence of Disintegration. Photographic evidence indicates that CDA materials and the positive controls disintegrated in seawater on time scales of months (Figure 1). Complete disintegration of the CDA films and fabrics and positive controls occurred on time scales of approximately three months. The positive controls generally disintegrated earlier than the CDA materials. The negative controls did not show any visual signs of disintegration throughout the 25-week incubation period.

Mass loss measurements confirmed the photographic evidence that the CDA and positive control materials disintegrated in seawater within months (Figure 2). For example, the CDA film (without a plasticizer) lost over $70 \%$ of its mass in 11 weeks in the mesocosm and disintegrated beyond the point of recovery after 13 weeks. The CDA fabric disintegrated beyond the point of recovery 2 weeks faster than the nonplasticized film, consistent with the 2.5-fold higher surface area:volume ratio of the fabric compared to that of the film (Table S1). The $510 \mu \mathrm{m}$ CDA foam initially lost mass at a rate comparable to those of the fabrics and films, aided by the rapid leaching of its plasticizer (Table S8). With time, the rate decreased, and the foam ultimately lost nearly $80 \%$ of its mass by 25 weeks (Figure 2 and Figure S2). The positive controls disintegrated the fastest among all of the materials tested. By 10 weeks, these positive control materials disintegrated to a point beyond handling and lost $60 \%$ of their mass. In contrast, the negative control materials did not disintegrate, with mass loss within the standard error of measurement $( \pm 5 \%)$. 


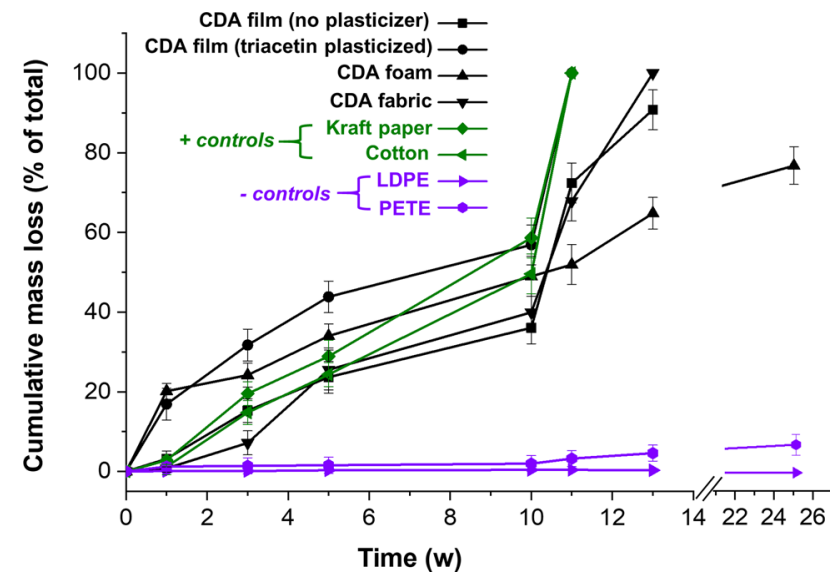

Figure 2. Measured mass loss of the CDA, positive control, and negative control materials incubated in a continuous flow seawater mesocosm over time. Error bars represent one standard error from the mean mass loss $(N=4)$.

Esterase and Cellulase Enzymatic Activity of Biofilm Communities. The rapid disintegration of the CDA materials is marked with increasing esterase and cellulase enzymatic activity, suggesting that the native community compositions are evolving with a metabolic capacity to degrade the CDA materials. The enzymatic approaches employed herein provide a general measurement of the in vitro hydrolytic capacity of the biofilm community. ${ }^{13,14}$ The deacetylation of CDA, as suggested through esterase activity measurements, manifested in the first weeks in the seawater mesocosm. Esterase activity profiled in the CDA materials and positive controls increased by an order of magnitude over the course of 10 weeks (Figure 3, Section S9, and Tables S3 and S4). Esterase activity was substantially higher in CDA materials and positive controls than in negative controls.

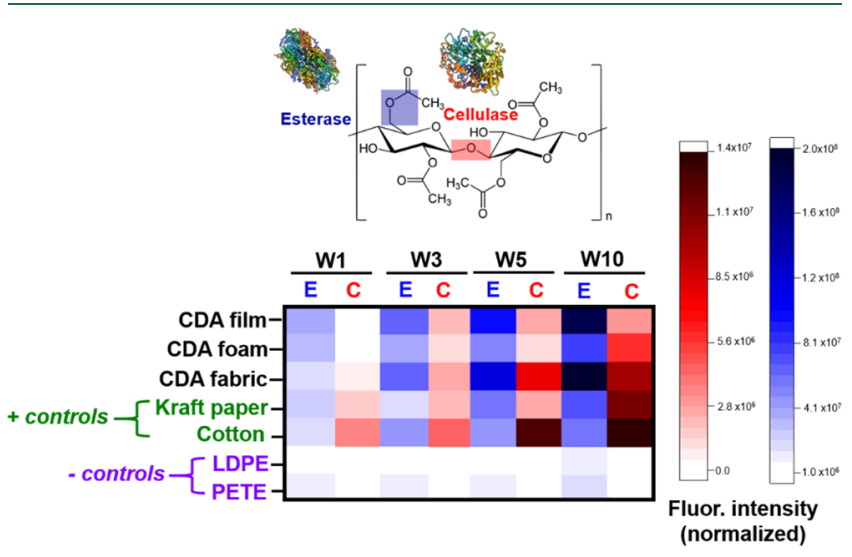

Figure 3. Esterase (blue) and cellulase (red) enzymatic activities [normalized to microbial biomass (Section S9)] profiled from natural biofilms growing on CDA materials and positive and negative controls in a continuous flow seawater mesocosm over weeks.

Cleavage of the cellulosic backbone of the CDA and positive control materials, as suggested by cellulase activity of the native microbial biofilms, increased by an order of magnitude over the course of the first 3 weeks in the mesocosm (Figure 3). The cellulase activity of the negative control, noncellulosic materials was substantially lower than that of CDA and positive control materials. Collectively, the assaying results suggest that the enzymatic activity of the native marine biofilms growing on CDA materials shifts toward esterase and cellulase degradative processes relative to the negative controls. An alternative interpretation of these data is that the cellulase and esterase activity is reflective of a general increase in metabolic activity, rather than shifts in metabolic pathways. This interpretation suggests that the specificity of the assays is limited (discussed in Section S9) and is consistent with the increased esterase activity in the cellulose-based positive controls. However, this alternative interpretation is inconsistent with the isotopic signatures of community respiration determined using shortterm bottle incubations and described in the following section. Independent of the mechanistic interpretation of the assaying results, the community growing on CDA and the positive controls exhibited notably higher enzymatic activity compared to that of the negative controls.

Tracking Biodegradation Using Natural Abundance $\Delta{ }^{14} \mathrm{C}$. The different precursors of CDA provide a unique opportunity to track its biodegradation over time using $\Delta^{14} \mathrm{C}$. CDA $\left[\Delta^{14} \mathrm{C}=-415 \pm 4 \%\right.$ (Table S5) $]$ is produced using a combination of modern cellulosic $\mathrm{C}\left[\Delta^{14} \mathrm{C}=98.0 \pm 3 \%\right.$ o (Table S5) $]$ and fossil acetyl C $\left[\Delta^{14} \mathrm{C}=-994 \pm 4 \%\right.$ (Figure S5 and Table S5)]. Provided the formulation, $\Delta^{14} \mathrm{C}$ can be robustly predicted (Figure S6). Therefore, the preferential degradation of specific components of CDA (e.g., cellulosic C vs acetyl C) may be reflected in the natural abundance $\Delta^{14} \mathrm{C}$ of $\mathrm{CDA}$ residue over time in the seawater mesocosm (as modeled in Figure S7). Moreover, respiration of $\mathrm{CDA}$ to $\mathrm{CO}_{2}$ during short-term bottle incubations would conceivably indicate which component of CDA is respired by marine microbes.

Despite substantial mass loss in the seawater mesocosm (Figures 1 and 2), the $\Delta^{14} \mathrm{C}$ of the $\mathrm{CDA}$ film and fabric residues remained relatively constant over time $\left[\Delta^{14} \mathrm{C}=-411\right.$ $\pm 4 \%$ (Figure $S 8$ and Table S6)], indicating equal loss of the acetyl and cellulose carbons (Figure S7). Degree of substitution measurements of the residues over time using nuclear magnetic resonance spectroscopy ( ${ }^{1} \mathrm{H}$ NMR) corroborated the $\Delta^{14} \mathrm{C}$ measurements (Table S7 and Figure S9). Relatively constant chemical compositions of the CDA residues, despite extensive degradation in the mesocosms (Figures 1-3), are consistent with previous reports in terrestrial systems. ${ }^{15,16}$ The exact mechanism governing the unvaried composition of CDA over time is unknown. A synergistic mechanism of esterase and cellulase enzymes that allows for rapid uptake of the resultant saccharide products is plausible and has precedent in soils and enzymatic approaches. ${ }^{13,14,17,18}$ This mechanism is also consistent with the enzymatic assaying, where esterase activity appeared weeks earlier than cellulase activity (Figure 3). Rapid turnover of the saccharide products would thus escape the temporal resolution required to track these intermediates isotopically and spectroscopically.

While the natural abundance $\Delta^{14} \mathrm{C}$ of the $\mathrm{CDA}$ residues in the mesocosm remained constant over time, shifts in the isotopic composition of seawater dissolved inorganic carbon (DI ${ }^{13} \mathrm{C}$ and $\mathrm{DI}^{14} \mathrm{C}$ ) during short-term (up to 6 days) bottle incubations with native microbial communities confirmed respiration of CDA (25 $\mu \mathrm{m}$ film without a plasticizer) to $\mathrm{CO}_{2}$. In all treatments, $\mathrm{DI}^{13} \mathrm{C}$ shifted toward more depleted values, indicative of microbial respiration of organic carbon (Figure 4). The shift in $\mathrm{DI}^{13} \mathrm{C}$ was notably more pronounced for the CDA and cellulose treatments than for the LDPE and seawater controls. Accordingly, respiration rates were an order of 


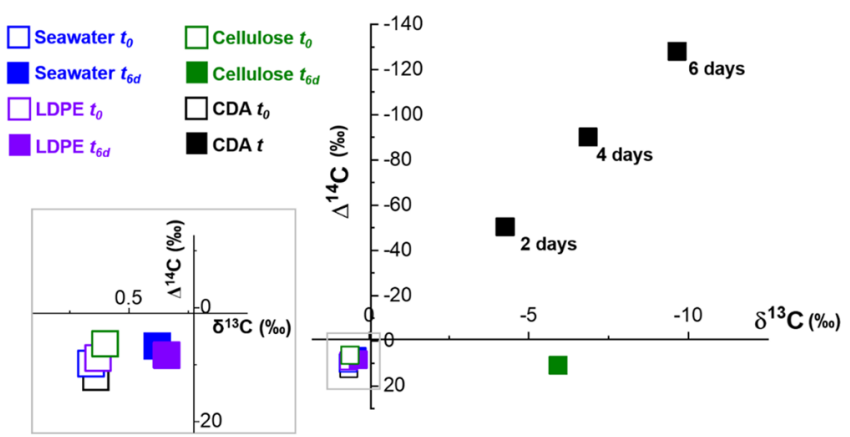

Figure 4. Tracking shifts in natural abundance $\mathrm{DI}^{13} \mathrm{C}$ and $\mathrm{DI}^{14} \mathrm{C}$ in seawater during short-term microbial respiration incubations. Initial $\mathrm{DI}^{13} \mathrm{C}$ and $\mathrm{DI}^{14} \mathrm{C}\left(t_{0}\right)$ are represented by empty symbols, and later time points $\left(t_{6 \mathrm{~d}}\right)$ are represented by filled symbols. Time points for the CDA $(25 \mu \mathrm{m}$ film without a plasticizer) treatment were collected at 2, 4, and 6 days (error bars are smaller than the symbols).

magnitude higher in the CDA $(14.0 \pm 1.1 \mu \mathrm{mol}$ of $\mathrm{C} /$ day $)$ and cellulose $[11.4 \mu \mathrm{mol}$ of $\mathrm{C} /$ day (Table S9) ] treatments than in the LDPE and blank seawater controls $(0.4 \mu \mathrm{mol}$ of $\mathrm{C} /$ day $)$. Throughout the 6-day incubation, nearly $3 \%$ of the CDA-C was respired to $\mathrm{CO}_{2}$ (Tables $\mathrm{S} 9$ and $\mathrm{S} 10$ and Figure S10). The CDA respiration series fit robustly into a first-order decay model $\left(R^{2}=0.99\right)$, with a first-order rate constant of $0.0046 \pm$ $0.0008 \mathrm{day}^{-1}$. This translates into a half-life of CDA of approximately five months under the conditions tested.

Only in the $\mathrm{CDA}$ treatment did the $\mathrm{DI}^{14} \mathrm{C}$ signature shift to more depleted values, reflecting the respiration of fossil, acetyl $\mathrm{C}$ in the CDA films (Figure 4). The mixed isotopic signature of the respired $\mathrm{CO}_{2}(-450 \pm 16 \%$ ) confirms that native marine microbes rapidly degrade the acetyl and cellulosic components of CDA. Furthermore, the slight preferential degradation of the fossil acetyl $\mathrm{C}\left[\mathrm{CDA}=-415 \pm 4 \%\right.$; respired $\mathrm{CO}_{2}=-450 \pm$ $16 \%$ (Table S9)] is consistent with the earlier onset of esterase activity compared to cellulase activity (Figure 3), supporting an overall mechanism in which deacetylation is the rate-limiting step of biodegradation. In the positive and negative control treatments, the $\mathrm{DI}^{14} \mathrm{C}$ signature remained constant with time, indicating that only modern $\mathrm{C}$ was respired, either from cellulose or from natural organic matter.

Implications for the Environmental Persistence of CDA. The environmental persistence of organic contaminants is a key component of risk assessment and regulatory frameworks, ${ }^{19,20}$ with some arguing that high persistence alone is sufficient for establishing regulations. ${ }^{21}$ However, applying this persistence-based regulatory framework to plastics remains a challenge due to incomplete understanding of the fates of these materials, particularly in the ocean. ${ }^{5}$ Our findings indicate that CDA-based materials are susceptible to both disintegration and degradation by marine microbes on time scales of months. These findings thus challenge the paradigm set by NOAA, governmental agencies, and advocacy groups that CDA-based materials persist in the ocean for decades. ${ }^{5,6}$ The time line of CDA biodegradation is inherently subject to variation depending on a number of factors not considered in this study. For example, increased surface area:volume ratios through disintegration and physical abrasion or photochemical alterations of CDA chemical compositions likely shorten lifetimes. ${ }^{22-25}$ Moreover, considering other fates of CDA-C, such as incorporation into biomass ${ }^{26}$ or the production of metabolites, ${ }^{27}$ would shorten lifetimes. In contrast, the lifetimes of thicker CDA materials with lower surface area:volume ratios in colder, darker, and nutrient-limited waters are likely longer. The balance of these factors in controlling the fate of CDA-based materials in the ocean should be explicitly determined in future research. While no one plastic type will likely meet all of the diverse and growing needs of consumers, these initial findings on the fate of CDA-based materials in the ocean, combined with previous reports in terrestrial and wastewater systems, ${ }^{7,8}$ represent a positive step toward identifying high-utility, biobased plastics with low environmental persistence.

\section{ASSOCIATED CONTENT}

\section{(I) Supporting Information}

The Supporting Information is available free of charge at https://pubs.acs.org/doi/10.1021/acs.estlett.1c00843.

CDA and control materials, surface area characterization, reagents and supplies used, continuous flow natural seawater mesocosm, biofilm liberation protocol, crystal violet staining protocol, natural abundance radiocarbon protocol, mass loss measurement protocol, enzymatic assaying protocol, compositional analysis protocols via NMR and plasticizer content analysis, and the protocol for respiration of CDA and control materials (PDF)

\section{AUTHOR INFORMATION}

\section{Corresponding Authors}

Michael G. Mazzotta - Department of Marine Chemistry and Geochemistry, Woods Hole Oceanographic Institution, Woods Hole, Massachusetts 02543, United States; - orcid.org/ 0000-0003-1797-1762; Email: mgm@whoi.edu

Collin P. Ward - Department of Marine Chemistry and Geochemistry, Woods Hole Oceanographic Institution, Woods Hole, Massachusetts 02543, United States; @ orcid.org/ 0000-0003-2979-0280; Email: cward@whoi.edu

\section{Author}

Christopher M. Reddy - Department of Marine Chemistry and Geochemistry, Woods Hole Oceanographic Institution, Woods Hole, Massachusetts 02543, United States; ๑ orcid.org/0000-0002-7814-2071

Complete contact information is available at: https://pubs.acs.org/10.1021/acs.estlett.1c00843

\section{Notes}

The authors declare no competing financial interest.

\section{ACKNOWLEDGMENTS}

M.G.M., C.M.R., and C.P.W. thank Eastman Chemical Co. and Woods Hole Oceanographic Institution (WHOI) for scientific and financial support. The authors are grateful to Gaurav Amarpuri, Brian Edwards, Mounir Izallalen, Sharmi Mazumder, Steve Perri, and Dawn Mason at Eastman Chemical Co. for their substantial intellectual contributions. The authors also appreciate helpful discussions and support from Carol Anne Clayson, Aran Mooney, Rick Galat, Natalie Renier, Justin Ossolinski, Taylor Nelson, Dave Bailey, Anna Walsh, and Danielle Freeman at WHOI, and Kathy Elder, Roberta Hansman, and Josh Burton at NOSAMS. Finally, the authors thank two anonymous reviewers and the associate editor for their constructive feedback during the review process. 


\section{REFERENCES}

(1) Jambeck, J. R.; Geyer, R.; Wilcox, C.; Siegler, T. R.; Perryman, M.; Andrady, A.; Narayan, R.; Law, K. L. Plastics waste inputs from land into the ocean. Science 2015, 347, 768-771.

(2) Geyer, R.; Jambeck, J. R.; Law, K. L. Production, use, and fate of all plastics ever made. Sci. Adv. 2017, 3, e1700782.

(3) Mohanty, A. K.; Vivekanandhan, S.; Pin, J.; Misra, M. Composites from renewable and sustainable resources: challenges and innovations. Science 2018, 362, 536-542.

(4) Araújo, M. C. B.; Costa, M. F. A critical review of the issue of cigarette butt pollution in coastal environments. Environ. Res. 2019, 172, 137-149.

(5) Ward, C. P.; Reddy, C. M. Opinion: We need better data about the environmental persistence of plastic goods. Proc. Natl. Acad. Sci. U. S. A. 2020, 117, 14618-14621.

(6) Arthur, C.; Baker, J.; Bamford, H. Proceedings of the International Research Workshop on the Occurrence, Effects and Fate of Microplastic Marine Debris, September 9-11, 2008; NOAA Technical Memorandum NOS-OR\&R-30.

(7) Buchanan, C. M.; Gardner, R. M.; Komarek, R. J. Aerobic biodegradation of cellulose acetate. J. Appl. Polym. Sci. 1993, 47, $1709-1719$

(8) Gardner, R. M.; Buchanan, C. M.; Komarek, R.; Dorschel, D.; Boggs, C.; White, A. W. Compostability of cellulose acetate films. J. Appl. Polym. Sci. 1994, 52, 1477-1488.

(9) Lynd, L.; Weimer, P. J.; van Zyl, W. H.; Pretorius, I. S. Microbial cellulose utilization: fundamentals and biotechnology. Microbiol. Mol. Biol. Rev. 2002, 66, 506-577.

(10) Danso, D.; Schmeisser, C.; Chow, J.; Zimmermann, W.; Wei, R.; Leggewie, C.; Li, X.; Hazen, T.; Streit, W. R. New Insights into the function and global distribution of polyethylene terephthalate (PET)degrading bacteria and enzymes in marine and terrestrial metagenomes. Appl. Environ. Microbiol. 2018, 84, n/a.

(11) Oberbeckmann, S.; Bartosik, D.; Huang, S.; Werner, J.; Hirschfeld, C.; Wibberg, D.; Heiden, S.E.; Bunk, B.; Overmann, J.; Becher, D.; Kalinowski, J.; Schweder, T.; Labrenz, M.; Markert, S. Genomic and proteomic profiles of biofilms on microplastics are decoupled from artificial surface properties. Environ. Microbiol. 2021, 23, 3099 .

(12) Zettler, E. R.; Mincer, T. J.; Amaral-Zettler, L. A. Life in the "Plastisphere": Microbial Communities on Plastic Marine Debris. Environ. Sci. Technol. 2013, 47, 7137-7146.

(13) Hoppe, H. G. Use of fluorogenic model substrates for extracellular enzyme activity (EEA). In Handbook of Methods in Aquatic Microbial Ecology, 1st ed.; CRC Press: Boca Raton, FL, 1993; pp 423-431.

(14) Sakai, K.; Yamauchi, T.; Nakasu, F.; Ohe, T. Biodegradation of cellulose acetate by Neisseria sicca. Biosci., Biotechnol., Biochem. 1996, 60, 1617-1622.

(15) Buchanan, C. M.; Dorschel, D.; Gardner, R. M.; Komarek, R. J.; Matosky, A. J.; White, A. W.; Wood, M. D. The influence of degree of substitution on blend miscibility and biodegradation of cellulose acetate blends. J. Environ. Polym. Degr. 1996, 4, 179-195.

(16) Bonanomi, G.; Incerti, G.; Cesarano, G.; Gaglione, S. A.; Lanzotti, V. Cigarette butt decomposition and associated chemical changes assessed by ${ }^{13} \mathrm{C}$ CPMAS NMR. PLoS One 2015, 10, e0117393.

(17) Komarek, R. J.; Gardner, R. M.; Buchanan, C. M.; Gedon, S. Biodegradation of radiolabeled cellulose acetate and cellulose propionate. J. Appl. Polym. Sci. 1993, 50, 1739-1746.

(18) Haske-Cornelius, O.; Pellis, A.; Tegl, G.; Wurz, S.; Saake, B.; Ludwig, R.; Sebastian, A.; Nyanhongo, G. S.; Guebitz, G. M. Enzymatic Systems for Cellulose Acetate Degradation. Catalysts 2017, $7,287$.

(19) United Nations. Stockholm convention on persistent organic pollutants. Publication 40214, 2001. https://treaties.un.org/doc/ Publication/MTDSG/Volume\%20II/Chapter\%20XXVII/XXVII-15. en.pdf (accessed 2021-11-27).
(20) European Agency for Safety and Health at Work (EU-OSHA). Regulation (EC) No 1907/2006 - Registration, Evaluation, Authorisation and Restriction of Chemicals (REACH). Publication 1907/ 2006, 2021. https://osha.europa.eu/en/legislation/directives/ regulation-ec-no-1907-2006-of-the-european-parliament-and-of-thecouncil (accessed 2021-11-27).

(21) Cousins, I. T.; Ng, C. A.; Wang, Z.; Scheringer, M. Why is high persistence alone a major cause of concern? Environ. Sci.: Processes Impacts. 2019, 21, 781-792.

(22) Chamas, A.; Moon, H.; Zheng, J.; Qiu, Y.; Tabassum, T.; Jang, J. H.; Abu-Omar, M.; Scott, S. L.; Suh, S. Degradation rates of plastics in the environment. ACS Sustainable Chem. Eng. 2020, 8, 3494-3511.

(23) Merlin, A.; Fouassier, J.-P. Photochemical investigations into cellulosic materials, IV. Photosensitized free radical generation in cellulose acetate and oligosaccharide compounds. Angew. Makromol. Chem. 1982, 108, 185-195.

(24) Hon, N. Photodegradation of cellulose acetate fibers. J. Polym. Sci., Polym. Chem. Ed. 1977, 15, 725-744.

(25) Song, Y. K.; Hong, S. H.; Jang, M.; Han, G. M.; Jung, S. W.; Shim, W. J. Combined effects of UV exposure duration and mechanical abrasion on microplastic fragmentation by polymer type. Environ. Sci. Technol. 2017, 51, 4368-4376.

(26) Popendorf, K. J.; Koblí̌̌ek, M.; Van Mooy, B. S. Phospholipid turnover rates suggest that bacterial community growth rates in the open ocean are systematically underestimated. Limnol. Oceanogr. 2020, 65, 1876-1890.

(27) Kujawinski, E. B. The impact of microbial metabolism on marine dissolved organic matter. Annu. Rev. Mar. Sci. 2011, 3, 56799.

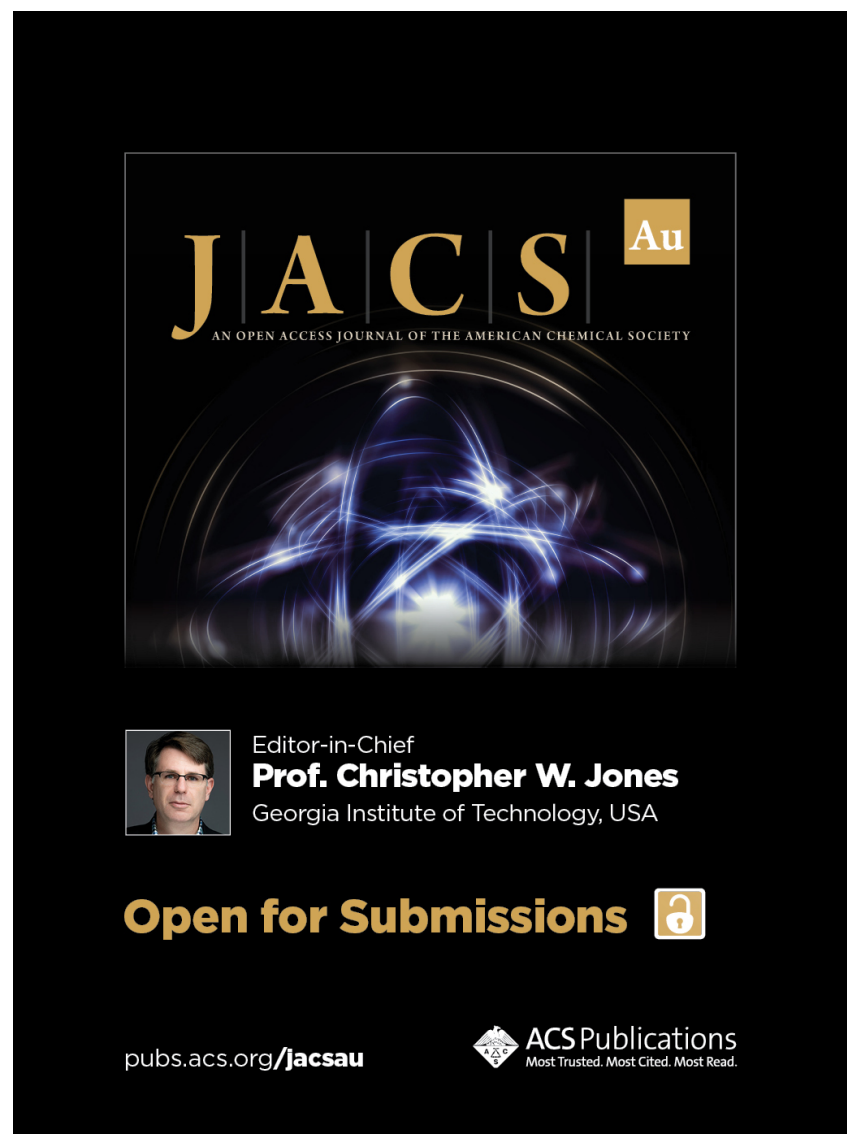

